\title{
CONDORCET : UNE THÉORIE DE LA CONNAISSANCE
}

On pourrait s'étonner que soit privilégié dans l'œuvre multiforme de Condorcet le thème de la théorie de la connaissance ${ }^{1}$. Mais si ce thème n'est explicitement traité que dans l'Esquisse d'un tableau historique des progrès de l'esprit humain, il ne sert pas moins de fil conducteur, de fondement à l'ensemble des domaines que Condorcet a abordés. Sa théorie de la connaissance serait, on l'a trop dit, un résumé hâtif, sans profondeur, des thèses qui, à partir de Locke et de Condillac, ont été adoptées et adaptées par la majorité des penseurs éclairés et les plus actifs des Encyclopédistes. Il est vrai que Condorcet participe sans remise en question, à l'esprit du temps. Encore est-il qu'il essaie précisément par sa théorie gnoséologique, de tenir ensemble, d'unifier les " moments " qui caractérisent cet esprit. Et c'est dans ce sens que la question de la connaissance est inséparable, dans son esprit, de celles du langage, du devenir de l'espèce humaine, du progrès de nos facultés; c'est en ce sens aussi que l'œuvre n'est ni disparate, ni compilatoire.

Condorcet considère comme beaucoup de ses contemporains que l'esprit de système est, avec le préjugé, l'obstacle majeur qui a ossifié la pensée philosophique; que la métaphysique, dans la mesure où elle se confond avec l'esprit systématique, n'aboutit qu'à des propositions vides, inutiles et, pire, dangereuses pour les progrès du savoir. Mais les systèmes ne sont nocifs que s'ils restent abstraits. La métaphysique,

1. Il faut préciser que l'expression "théorie de la connaissance " n'appartient pas au vocabulaire de Condorcet; elle apparaît en Allemagne dans des ouvrages qui se situent dans la lignée de Kant. On peut citer de Georg KRUG, Erkenntnislehre oder Metaphysik, 1808 ; et de Karl Leonhard REINHOLD, Theorie des menschlichen Erkenntnisvermögens und Metaphysik, 1832-1834. L'expression a, semble-t-il, été répandue à partir de la publication du texte d'Eduard ZELLER, Über Aufgabe und Bedeutung der Erkenntnistheorie, 1862. Reste qu'avant l'emploi de l'expression, chaque système philosophique a dû décider de la question de savoir comment la pensée humaine se représente des objets et peut aboutir à une connaissance. C'est en ce sens qu'on peut utiliser la notion de " théorie de la connaissance ", avant la lettre. 
asile de l'ignorance jusqu'ici, est susceptible de recevoir un sens nouveau : elle devient la science qui, à partir d'observations contrôlées, permet de connaître les faits généraux et les lois constantes que présente le développement des facultés humaines. La métaphysique ne peut devenir science qu'en découvrant sa propre vérité si longtemps occultée : elle est la science de l'homme. Le projet de Condorcet est donc bien de constituer un système : à la diaspora des savoirs, il oppose la nécessité de construire un tableau (système) raisonné.

Les premières lignes de l'Esquisse peuvent apparaitre comme un rappel expéditif de la théorie sensualiste, empiriste, de la connaissance, inspirée tout à la fois de Locke, Condillac, Rousseau et du Discours préliminaire de d'Alembert à l'Encyclopédie.

"L'homme naît avec la faculté de recevoir des sensations; d'apercevoir et de distinguer dans celles qu'il reçoit les sensations simples dont elles sont composées, de les retenir, de les reconnaître, de les combiner; de comparer entre elles ces combinaisons; de saisir ce qu'elles ont de commun et ce qui les distingue; d'attacher enfin des signes à tous les objets, pour les reconnaître mieux, et en faciliter des combinaisons nouvelles $"{ }^{2}$.

Rappelons ici que le postulat fondamental de la théorie empiristesensualiste est que toutes les connaissances humaines (tant au niveau de l'individu que de l'espèce) s'originent dans la sensation et en dérivent selon un processus génétique. La sensation, élément premier, est précisément l'impression provoquée sur un organe sensible par un objet extérieur. Nous ne connaissons pas, stricto sensu, l'objet extérieur en lui-même mais l'effet qu'il produit en nous. Ainsi dans l'Essai sur l'origine des connaissances humaines, Condillac peut affirmer que " nous ne sortons point de nous-mêmes; ce n'est jamais que notre propre pensée que nous apercevons ". Condorcet, sur ce point, ne suit pas Condillac. Un empirisme conséquent implique à ses yeux l'existence hors de nous des objets extérieurs, puisque précisément ils sont cause de nos sensations. La question de savoir si nous sommes enfermés dans un monde de représentations purement subjectives lui semble relever d'un esprit métaphysique fourvoyé. L'homme donc, au premier état de son existence (qu'il s'agisse de l'enfance de l'individu ou

2. CONDORCET, Esquisse d'un tableau historique des progrès de l'esprit humain, Paris, Éditions sociales, 1971 , p. 75 . 
de celle de l'humanité), éprouve différentes sensations qui sont la source de ses premières pensées.

Selon Condorcet, les plaisirs et les douleurs ne sont pas - et en cela il se sépare de Condillac - des sensations élémentaires mais des impressions dérivées, s'il est vrai que l'ordre du sentir, de l'affectif, est plus complexe que celui du sentir pur.

Des sensations ne peuvent surgir des connaissances que si interviennent des opérations de l'esprit que Condillac caractérise par le terme général de réflexion. Ces opérations décrites avec minutie dans l'Essai sur l'origine des connaissances humaines sont d'abord la perception ou aperception. Dans la perception, l'âme éprouve parfois avec conscience (il s'agit alors de l'opération d'attention), parfois sans conscience actuelle ${ }^{3}$. La conscience se définit par le degré de vivacité de l'impression qui accapare et focalise l'attention. Ensuite, à partir de l'attention, une perception passée peut se reproduire en l'absence de l'objet qui l'a provoquée : ainsi s'engendre la mémoire. Condillac distingue l'imagination (qui est la possibilité de revivre une impression vécue) et la mémoire elle-même (qui a la capacité de se souvenir de l'impression sans la revivre effectivement). Le souvenir est plus proche de l'idée abstraite que l'image. La mémoire devient réminiscence quand elle reconnaît expressément les impressions déjà éprouvées. Dès l'opération de la mémoire apparaît la fonction du signe : car elle ne joue plus sur les impressions issues des objets, mais sur leurs substituts. C'est encore de la théorie condillacienne des signes que Condorcet s'inspirera. C'est grâce aux signes, en effet, qu'il est possible de reconnaître les objets et d'effectuer des combinaisons nouvelles. Le type de signe que Condillac privilégie ${ }^{4}$ est le signe dit d'institution ou arbitraire; s'il n'est pas nécessaire à l'imagination parce que la vue de l'objet suffit à réveiller l'impression, il est indispensable à la genèse et à l'exercice de la mémoire. En possession de signes arbitraires, l'âme se libère de la dépendance qui l'asservissait à l'impression pure. Dès lors il devient possible d'appliquer l'attention aux signes eux-mêmes : c'est l'opération proprement réflexive qui n'est pas un pouvoir inné de l'esprit ${ }^{5}$ mais bien au contraire un résultat de la combinaison d'opérations élémentaires. C'est au pouvoir de la réflexion qu'il appartient

3. Pour Condillac, toute impression est consciente; si elle ne l'était pas, elle ne pourrait pas engendrer une connaissance. Certaines impressions deviennent inconscientes parce qu'on les a oubliées. Condorcet suit la pensée condillacienne : il s'agit de mettre en question la théorie leibnizienne des " petites perceptions " inconscientes et, plus profondément, sa théorie des idées innées.

4. Condillac admet l'existence de signes accidentels et de signes naturels, mais ils n'ont pas de fonction dans le mécanisme de la connaissance. Cf. Essai, l re partie, chap. IV.

5. Sur ce point, Locke hésitait. 
désormais d'effectuer les opérations de distinction (on considère les idées séparément par analyse), d'abstraction (on ne retient que les caractères communs à un groupe d'idées), on compare, on compose, on décompose, on combine, en un mot, on pense. Il en résulte que les idées générales et abstraites sont des êtres de raison, accompagnées de signes appropriés, elles n'ont de réalité que nominale.

De l'empirisme condillacien Condorcet retient que la pensée humaine procède naturellement par combinaisons et que lorsqu'elle s'explicite elle-même dans le discours de la philosophie, c'est-à-dire lorsqu'elle se réfléchit, elle procède selon une méthode qui reflète le processus naturel de son développement et de son exercice. La méthode d'analyse est celle même de la pensée, puisque comprendre c'est composer, décomposer, recomposer. L'analyse est doublement génétique : elle met au jour les mécanismes de l'évolution de l'esprit individuel et de l'esprit à l'œuvre dans l'espèce. La méthode génétique est aussi à l'inverse, ce mouvement régressif qui permet de retrouver les éléments les plus simples de la connaissance. Enfin, la méthode analytique est heuristique : elle permet d'instaurer de nouvelles combinaisons. On comprend que si pour Condillac, et après lui Condorcet, le langage est lui-même de nature analytique (la langue décompose et recompose les éléments de la pensée), alors il est nécessaire de constituer une langue des calculs, une langue universelle.

Condorcet reprend une idée qu'on peut dire triviale au XviII siècle : il y a un rapport des langues aux gouvernements. Autant il s'intéresse peu à la question de l'origine du langage et des langues (l'homme est par nature sociable, il est habité par le besoin de communiquer et cela suffit ${ }^{6}$ ), autant il est soucieux de faire apparaître que chaque état de langue est lié à un état des facultés de l'esprit, lui-même relié à tel niveau du développement économique, social et politique ${ }^{7}$. Le langage est inséré dans une histoire globale, celle de l'espèce, et les histoires diversifiées des sociétés. Il y a une histoire des langues parce qu'il y a indissociablement une histoire de la pensée et plus profondément une histoire de l'espèce.

C'est encore dans la lignée de Condillac que Condorcet admet qu'il y a à la fois une décadence des langues et une possibilité de les perfectionner. La décadence signifie qu'en évoluant une langue perd sa

6. C'est la même raison: l'homme est naturellement sociable, qui explique que Condorcet ne soutient pas une théorie de l'origine de la société fondée sur un contrat.

7. La question serait de savoir si on peut, dans ce réseau complexe de corrélations, " isoler " un type de causalité qui serait déterminant. Condorcet s'est posé la question ; sa réponse semble bien être que si causalité déterminante il y a, elle est variable selon les époques ou périodes de l'histoire humaine. 
pureté originelle ; elle perd son caractère "figuré ». Dans l'état figuré où règne la métaphore, le signe est image, il est au plus proche de la sensation, donc de l'élément premier du connaître. La décadence est éloignement par rapport à la nature; le signe figuré fait place au signe arbitraire. Mais cette décadence indique et rend possible indissociablement un progrès en rationalité. Ce double mouvement - décadence et progrès - habite sous une forme paroxystique la réflexion de Rousseau sur l'origine des langues et des sociétés. Condillac affirme la nécessité d'accélérer le progrès des langues par l'instauration d'une langue universelle qui correspondrait exactement au fonctionnement de l'esprit : à chaque idée serait attaché un signe; une syntaxe est à constituer qui reproduirait les opérations mentales de dissociation, de combinaison... que l'analyse a mises au jour et qui sont elles-mêmes de nature analytique.

Un projet semblable est repris dans l'Esquisse sous forme sans doute plus ambitieuse. Condillac en effet semble avoir un souci essentiellement gnoséologique : la langue universelle est indispensable au progrès des sciences. Condorcet ne sépare pas l'instauration d'une langue universelle d'une perspective téléologique. Dans la $\mathrm{X}^{\mathrm{e}}$ période de l'Esquisse (qui considère les progrès futurs de l'esprit humain), il est affirmé que l'analyse des principes des sciences physiques et morales est toute récente, que l'analyse des facultés intellectuelles est encore imparfaite. Les progrès de l'analyse de nos facultés, de l'analyse des principes scientifiques et le perfectionnement des langues obéissent aux mêmes lois qui sont celles de la rationalité.

On aboutit à une série d'équations :

À chaque objet ou collection d'objets correspond une idée générale. Les opérations de l'esprit combinent les idées. Les idées et les opérations constituent la structure et le fonctionnement de l'esprit humain universel. Si à chaque idée correspond un signe et si les opérations se traduisent dans une syntaxe, alors l'esprit humain universel, convenablement analysé dans sa nature et ses productions, doit pouvoir posséder une langue universelle. "On connaîtrait le signe en même temps que l'objet, l'idée, l'opération qu'il désigne " ${ }^{8}$.

Cette langue à constituer est comme l'algèbre. Elle aurait pour avantage de pouvoir être pratiquée par tous; ainsi Condorcet pense pouvoir éviter le danger, souvent souligné par lui, qui serait inhérent à l'utilisation d'une langue savante par quelques initiés et que le vulgaire ne saurait comprendre. Les langues savantes divisent le corps

8. CONDORCET, op. cit. supra n. 2, p. 280. 
social en deux classes et entretiennent les préjugés qui maintiennent le peuple dans l'ignorance .

Un problème doit ici être esquissé : la langue universelle est-elle vouée à n'être qu'une langue écrite ou peut-elle être parlée ? La question n'est pas nouvelle ${ }^{10}$ lorsque Condorcet écrit l'Esquisse; il propose une solution qui peut paraître expédiée :

" Ainsi les hommes qui connaîtraient ces signes, la méthode de les combiner et les lois de leur formation, entendraient ce qui est écrit dans cette langue et l'exprimeraient avec une égale facilité dans la langue commune du pays $"$ ".

Qu'on puisse exprimer la langue universelle dans la langue commune sous-entend qu'en dépit de leur diversité empirique les langues obéissent aux mêmes lois de signification et de combinaison, qu'il y a une structure universelle dans toute langue. Disons que toute langue est logique puisqu'elle exprime le réel qui est fondamentalement réductible en éléments saisissables par la raison. Dans quel système d'écriture pourra-t-on exprimer la langue universelle? Selon Condorcet qui, sur ce point encore, épouse les idées reconnues de son temps, l'écriture alphabétique couronne les autres systèmes (l'écriture par peinture des objets, l'écriture hiéroglyphique) parce qu'elle est capable d'attacher un signe conventionnel à un son; l'écriture alphabétique est analytique. Est-elle donc la forme d'expression privilégiée pour transcrire la langue universelle? Le rêve de Condorcet s'annonce ici : la langue universelle a bien rapport à l'écriture alphabétique, mais ce rapport reste figuré. La langue universelle devrait avoir son.écriture propre; elle aurait un statut pensable par analogie avec l'algèbre. Mais il ne peut s'agir que d'une analogie. Comment en effet écrire dans la transparence radicale la nature même de l'esprit?

Quoi qu'il en soit, la langue universelle exotérique par excellence résulte des progrès des Lumières et accélère ce progrès. Elle s'oppose aux langues occultes qui perpétuent superstitions, despotismes et dogmatismes. Susceptible d'être comprise par tous, elle suppose l'égalité des hommes. Elle est inséparable, politiquement, de la démocratie. Le souci gnoséologique de Condillac apparaît ici circonscrit, étroit. Dans l'instauration de la langue universelle fondée sur l'analyse qu'elle reflète et promeut, Condorcet aperçoit la possibilité d'unir la connais-

9. Par exemple, le latin pratiqué par l'Église.

10. Leibniz avait rencontré cette question : comment penser le rapport d'une langue caractéristique universelle et d'une langue parlée?

11. CONDORCET, op. cit. supra n. 2, p. 279. 
sance vraie, la conscience de soi et l'accès au bonheur. L'analyse permet, en effet, de mettre au jour qu'étant donnés tels éléments, tels types de combinaisons " en nombre presque infinis" sont possibles ${ }^{12}$. Dès lors, l'analyse et la langue universelle peuvent servir de «base à l'art social " et politique. Il s'agit désormais de trouver la meilleure combinaison possible qui réalise la liaison - toujours rêvée par la philosophie - de la vérité et du bonheur.

Retenons que la combinatoire ne peut réaliser n'importe quoi, puisque des lois de structure et de fonctionnement existent. Mais l'art de combiner permet à l'homme des Lumières d'opposer le hasard à luimême puisqu'il connaît les lois de l'art. Il appartient désormais au philosophe d'utiliser l'analyse - cette forme suprême du calcul des possibles - que la métaphysique avait indûment abandonnée à Dieu.

En possession des lois de l'analyse et d'une langue adéquate, la philosophie ne se limite pas à l'ambition raisonnable d'opposer lè hasard à lui-même. $\mathrm{Si}$, dans un tableau, on peut enclore les faits connus et classés, si on connaît les lois de passage d'une case à l'autre et l'ordre de classement, il sera possible :

1) D'avoir une vue synoptique du devenir de l'espèce humaine.

2) De combler des lacunes dans nos connaissances sur les premières périodes de l'humanité ${ }^{13}$. L'histoire ici se confond avec l'usage raisonné d'hypothèses elles-mêmes fondées sur des observations.

3) De prévoir et donc de déterminer pour le futur des actions raisonnables.

Il apparaît que l'histoire est le lieu où se développe l'espèce dans un processus progressif que Condorcet ne met pas en question; qu'elle est l'objet de la réflexion du philosophe en ce qu'il la constitue comme objet de connaissance scientifique; qu'elle est le lieu et l'objet même de la philosophie s'il est vrai que la philosophie est le développement de la rationalité.

L'empirisme de Condorcet n'est pas une simple reprise obéissante, timide, du sensualisme de son siècle. S'il ne modifie pas cette théorie de la connaissance en profondeur, il l'exploite comme un ensemble de principes unificateurs qui permettent de rendre intelligible le devenir de l'espèce. On pourrait risquer de dire que dans la mesure où Condorcet est sans cesse soucieux d'unir expérience et raison, obser-

12. Ibid., p. 271.

13. Ibid., p. 87. 
vation et vérification, analyse et prévision, il représente une des figures de l'empirisme rationnel.

On a dit de son œuvre qu'elle était éclectique. Mais au XviII siècle, l'éclectisme n'est pas un ramassis de fragments de vérités cueillies ici ou là dans d'incompatibles systèmes. Il est juste de dire que Condorcet est un philosophe éclectique au sens où Diderot a défini ce terme dans l'Encyclopédie: l'éclectisme est la philosophie qui, fondée sur l'expérience et l'usage critique de la raison, caractérise les temps nouveaux.

Michèle Crampe-Casnabet, École normale supérieure, Fontenay-aux-Roses. 